\title{
EDITORIAL
}

\section{What just happened (and keeps on happening)?: The experience of COVID-19 in Canadian pharmacy practice and education}

\author{
Zubin Austin \\ University of Toronto, Canada
}

Correspondence

Prof Zubin Austin

Leslie Dan Faculty of Pharmacy

University of Toronto

144 College Street

Toronto ON

M5S 3M2

Canada

zubin.austin@utoronto.ca

\begin{abstract}
The Canadian experience in managing the first wave of COVID-19 highlights the value of unified political leadership, belief in experts, evidence-informed decision making and social cohesion. Though far from perfect, Canada's response was built upon a foundation of universally accessible health care and faith in public health guidance. Community pharmacy has been an integral part of care delivery during COVID-19. As a second or third wave evolves, it is essential to learn from previous experiences to better prepare for what comes next.
\end{abstract}

If we had not all lived through it, we would not have believed it possible. The story of COVID-19, and the way it has fundamentally changed our professional and personal lives, continues to evolve. Within pharmacy practice, the initial waves of COVID-19 have triggered significant discussion, research, and speculation regarding the future of the profession (Elbeddini et al., 2020; Hayden \& Parkin, 2020; Visacri, Figueredo \& de Mendonca Lima, 2020). In Canada, pharmacists were recognised as essential workers, providing service and care to an increasingly distraught public (Elbeddini, Hooda, \& Wang, 2020). Recent research has quantified the significant contributions made by pharmacists to the health and well-being of their communities during an unprecedented time of social stress (Goff et al., 2020; Gregory \& Austin, 2020). A central finding of this research has been that as primary care systems in Canada closed down or shifted to virtual care models, community pharmacies emerged as one of the most important sources of information, personal protective equipment, medical supplies, and in-person care available to average citizens (Gregory \& Austin, 2020). In particular, as misinformation regarding miracle cures such as hydroxychloroquine or injectable bleach was being circulated, or as medication stockpiling and drug shortages threatened the integrity of the pharmaceutical supply chain, community pharmacists emerged as crucial, impartial, and accessible sources of information and guidance for the general public (Austin \& Gregory, 2020; Gregory \& Austin, 2020).

Pharmacy education in Canada has also had to rapidly evolve in order to meet the constraints and realities of current conditions. Aligning pharmacy education with FIP Workforce Development Goals has proved challenging; across the country, innovative practices have proliferated as university-based and clinical educators worked to ensure continuity and support for students and trainees at all levels (International Pharmaceutical Federation [FIP], 2016). 


\section{FIP Workforce Development Goal \#1: Academic capacity}

Across Canada, there are ten schools of pharmacy (eight English-language, two French-language), most of which are located within medical-doctoral university settings with access to academic health sciences centres (Austin \& Ensom, 2008). Compared to many other countries, there are relatively few pharmacy education programmes available for Canadians; as a result, each year more than $30 \%$ of all newly registered pharmacists across the country are international pharmacy graduates (IPGs, individuals who received their initial pharmacy education/ training outside Canada) (Austin \& Rocchi-Dean, 2007; Austin \& Galli, 2003). Prior to the pandemic, there were concerns regarding the adequacy of academic capacity to meet Canadian pharmacy needs (Austin \& Ensom, 2020). There have been no reported closures of pharmacy programmes or reductions in class sizes since the start of the pandemic. All Canadian pharmacy programmes have shifted to blended teaching/learning/assessment models with most coursework being delivered online using diverse synchronous or asynchronous platforms (e.g. Zoom, Microsoft Teams, or Blackboard Collaborate), with some live (in-person), skills-based or laboratory-based teaching and assessment required for certain courses (e.g. injections training, physical assessment, or pharmaceutics/ compounding). Bridge training programmes designed to support IPGs in meeting Canadian practice standards have also continued to be delivered in a virtual manner.

A core feature of Canadian pharmacy education has been its emphasis on interpersonal and inter-professional skills training, using patient simulation techniques such as Objective Structured Clinical Examinations (OSCEs) (Austin \& Ensom, 2008; Shirwaikar, 2015). Social distancing requirements have made it problematic to deliver such programming in a face-to-face manner. Across the country, educators have developed innovative online methods of simulating virtual care provision online, and this has led to new methods for assessing clinical and communication skills through video-recorded online interactions with simulated patients (Park \& Min, 2020). Overall, the pandemic has not resulted in any adverse impacts on academic capacity in pharmacy education and has led to a proliferation of innovative online educational practices that are likely to continue after pandemic conditions end.

\section{FIP Workforce Development Goal \#2: foundation training}

Canadian pharmacy education relies heavily upon in-service foundation training in the form of early pharmacy practice experiences (in years one and two) and advanced pharmacy practice experiences (APPE clinical rotations in year four) (Austin \& Ensom, 2008). Approximately $25-30 \%$ of the entire pharmacy degree in Canada is based on foundation training in community or hospital clinical settings, under the supervision of trained preceptors (Austin \& Ensom, 2008). With the declaration of the pandemic in mid-March 2020, all foundation training in most health professions in Canada was temporarily suspended as a way of maintaining social distance (Calhoun \& Yale, 2020; Louiselle et al., 2020). Students in mid-rotation were immediately withdrawn from all clinical placement sites. Students who had not completed all rotations required for their degree were informed they would not be proceeding to clinical sites. Given the extraordinary circumstances, universities declared an emergency situation and permitted all students to graduate with their degrees even if they had not completed all degree-required clinical rotations.

Universities moved quickly to identify different foundational training opportunities for students in the subsequent academic years of the programme who would also experience cancelled clinical rotations. While these could not necessarily replace traditional clinical rotations, they offered students alternative, meaningful opportunities for the application of clinical knowledge and skill. Virtual care rotations (e.g. online medication reviews with patients, telephone-based consultations etc.) rapidly evolved as community pharmacy practice pivoted to online care delivery. Additional non-clinical rotations (e.g. focused on pharmacy education or practice research) were developed by faculty members to allow students to meet degree-requirements for rotations in a nontraditional manner. Between virtual care options and these additional non-clinical rotations, all students were able to secure the required number of foundation training rotations required for graduation. It is anticipated that once pandemic conditions subside, most of these additional types of rotations will continue to be offered, providing students with greater options and opportunities to explore diverse career interests and pathways.

\section{FIP Workforce Development Goal \#8: working with others}

The Canadian health system is characterised by a high degree of inter-professional collaboration and a relatively open approach to scopes of practice related to core activities such as prescribing. Inter-professional collaboration is a specific registration competency that is assessed for all healthcare professionals seeking licensure in Canada (Suter et al., 2009; Baker \& Durham, 2013; Wood et al., 2009). In the early days of the pandemic, most primary care settings (e.g. family doctors' offices, health teams, and clinics) were closed to live appointments and shifted 
to virtual care options (e.g. online or telephone-based consultations). Since collaboration in primary care between pharmacists and other healthcare professionals has historically been primarily through virtual means, there were few reported disruptions in regular activities (Chen \& de Almeida Neto, 2007). Inter-professional education was also relatively quickly moved to virtual platforms. Formal education sessions (e.g. workshops) that had historically been offered live were moved to Zoom or other virtual platforms relatively quickly. Assessment of inter-professional competencies was also adapted to virtual formats without significant changes required. Anecdotally, the general shift towards virtual care provision across primary care in Canada appeared to actually expand the need for collaboration, and this was reflected in greater opportunities for students to contribute to primary care through (for example) virtual medication reconciliation, virtual medication/health education sessions, and virtual team meetings. As comfort has grown with these formats in primary care (for both healthcare professionals and patients), it is anticipated that virtual primary care will continue to expand significantly post-pandemic and that opportunities for inter-professional collaboration and education will similarly expand in the years to come.

\section{FIP Workforce Development Goal \#9: Continuing professional development strategies}

Initial rapid-response research at the outset of the pandemic highlighted the need for all pharmacists (but community pharmacists in particular) to rapidly pivot and shift to new practice models emphasising technology and virtual care provision. The immediate learning needs of the entire workforce for training and skills development were significant. Unfortunately, the capacity of the education and professional development sector to meet this sudden and unprecedented need was insufficient and many pharmacists reported a somewhat chaotic and difficult first few months of the pandemic as they all struggled to cope and adapt without a coherent profession-wide Continuing Professional Development (CPD) strategy in place. As the pandemic evolved, CPD providers were able to more rapidly and fully exploit the potential of online and virtual education systems and a proliferation of webinars, online workshops, and other CPD options became available and widely utilised to support pharmacists' practice. In general, CPD delivery during this time was reactive and responsive, rather than strategic: specific sessions focused on (for example) medical information updates regarding the role of hydroxychloroquine in COVID-19 management or skills-building related to virtual medical reconciliation. As the pandemic progressed and experience with online CPD continued to grow, professional associations and regulators began to plan more tactically for workforce development and exploit significant advantages of online CPD (e.g. asynchronous delivery options, self-paced learning opportunities etc.). Anecdotally, it appears as though pharmacists are accessing online CPD more extensively than they have ever accessed live CPD, and that the focused/targeted nature of this online CPD has been of practical value in skills-building for pandemicrelated needs. Further research is required to corroborate this observation. Currently, there is no profession-wide process or dialogue focused on developing a more coherent CPD strategy for Canadian pharmacists, and the ad-hoc, reactive approach that dominated the early days of the pandemic continues to operate.

\section{Where do we go from here?}

Most Canadian pharmacists are justifiably proud of the way their profession has performed during the pandemic. While there have been strengths and challenges in the way academic pharmacy in Canada has responded to the pandemic, there has been general satisfaction expressed by students and faculty members in the way education and training programmes have pivoted to the new realities introduced by COVID-19.

It is unclear what lasting changes in pharmacy education in Canada will be the legacy of COVID-19. The relative ease with which most pharmacy education (including CPD) has pivoted to online delivery and assessment suggests this will become a somewhat permanent feature into the future. Courses and content that can be offered online (through synchronous or asynchronous delivery) will likely continue in this format in the future. This means that pharmacy education in Canada may become far more accessible than ever before, as significant geographical barriers to access become less problematic. For a large, sparsely populated country like Canada, this has enormous potentially positive implications (Austin \& Ensom, 2008). Further, as experience with virtual and non-traditional models of foundation training and clinical rotations have grown, these too will likely become permanent within the profession.

A significant issue remains unresolved in pharmacy education and practice: licensure examinations. Since 2000, the Pharmacy Examining Board of Canada has required a 16-station OSCE as part of the entry-to-practice assessment of competency recognised by regulatory bodies. Due to social distancing requirements - and the need to ensure examination security - as of late November 2020, it has not yet been possible to organise a 
live, in-person OSCE or to develop an online equivalent. As a result, many students of the graduating class of 2020 (who should have been able to complete this registration requirement in May 2020) are still waiting to do so and are therefore unlicensed as pharmacists. Beyond the personal toll of this situation, it is causing problems in the workforce as the next generation of pharmacists cannot start practicing independently and the class of 2021 awaits word on how OSCEs will be managed for them. Ongoing discussions between regulators, educators, professional associations, and employers continue, but as of this writing, no resolution to this issue has been announced.

What comes next? While we are in the middle of digesting the question of 'what just happened?' it can be difficult to plan for the future. If there is any lesson to be drawn from COVID-19, it is that contingency planning is not simply for emergencies but something that must be part of our everyday life going forward. As a profession, pharmacy has many strengths to see us through whatever comes next: a strong educational system, dedicated leadership interested in public health, a practitioner base committed to science and evidence, and high levels of trust with the public. This foundation will be crucial, but it is not necessarily sufficient to see us through the months and years ahead.

\section{References}

Austin, Z., \& Ensom, M.H.H. (2008). Education of pharmacists in Canada. American Journal of Pharmacy Education, 72(6). https://doi.org/10.5688/aj7206128

Austin, Z., \& Galli, M. (2003). Assessing communicative competency of international pharmacy graduates in Ontario, Canada. Journal of Social and Administrative Pharmacy, 20(6), 225-231

Austin, Z., \& Gregory, P. (2020). Resilience in the time of pandemic: the experience of community pharmacists during COVID-19. Research in Social and Administrative Pharmacy (EPub ahead of print). https://doi.org/10.1016/j.sapharm.2020.05.027

Austin, Z., \& Rocchi-Dean, M. (2007). Bridging education for foreign trained professionals: the international pharmacy graduate (IPG) program in Canada. Teaching in Higher Education, 11(1), 19-32. https://doi.org/10.1080/13562510500400081

Baker, M.J., \& Durham, C.F. (2013). Interprofessional education: a survey of students' collaborative competency outcomes. Journal of Nursing Education, 52(12), 713-718

Calhoun, K.E., \& Yale, L.A. (2020). The impact of COVID-19 on medical student surgical education: implementing extreme pandemic response measures in a widely distributed surgical clerkship experience. The American Journal of Surgery, 220(1), 44-77. https://doi.org/10.1016/i.amjsurg.2020.04.024
Chen, T.F., \& de Almeida Neto, A.C. (2007). Exploring elements of interprofessional collaboration between pharmacists and physicians in medication review. Pharmacy World \& Science, 29(6), 574-576. https://doi.org/10.1007/s11096-007-9130-2

Elbeddini, A., Prabaharan, T., Almasalkhi, S., \& Tran, C. (2020). Pharmacists and COVID-19. Journal of Pharmaceutical Policy and Practice, 13(36). https://doi.org/10.1186/s40545-020-00241-3

Elbeddini, A., Hooda, N., \& Wang, L. (2020). Role of Canadian pharmacists in managing drug shortages amid the COVID-19 pandemic. Canadian Pharmacists Journal, 153(4), 198-203. https://doi.org.10.1177/1715163520929387

Goff, D.A., Ashiru-Oredope, D., Cairns, K.A., Eljaaly, K., Langford, B.J., Messina, A.P., \& Saad, T. (2020). Global contributions of pharmacists during the COVID-19 pandemic. Journal of the American College of Clinical Pharmacy (EPub ahead of print). https://doi.org/10.1002/jac5.1329

Gregory, P., \& Austin, Z. (2020). COVID-19: How did community pharmacists get through the first wave? Canadian Pharmacists Journal, 153(5), 243-251. https://doi.org/10.1177/17151635209 $\underline{45741}$

Hayden, J.C., \& Parkin, R. (2020). The challenges of COVID-19 for community pharmacists and opportunities for the future. Irish Journal of Psychological Medicine, 37(3), 198-203. https://doi.org/10.1017/ipm.2020.52

International Pharmacy Federation (FIP) (2016). Pharmaceutical Workforce Development Goals. Available at: https://www.fip.org/ www/streamfile.php?filename=fip/PharmacyEducation/2016 rep ort/2016-11-Education-workforce-development-goals.pdf

Louiselle, K., Elson, E.C., Oschman, A., \& Duehlmeyer, S. (2020). Impact of COVID-19 on pharmacy learners and preceptors. American Journal of Health-System Pharmacy, 77(14), 1097-1099. https://doi.org/10.1093/ajhp/zxaa127

Park, J.Y., \& Min, J. (2020). Exploring Canadian pharmacy students' e-health literacy: a mixed methods study. Pharmacy Practice, 18(1). https://dx.doi.org/10.18549/pharmpract.2020.1.1747

Shirwaikar, A. (2015). Objective structured clinical examination (OSCE) in pharmacy education - a trend. Pharmacy Practice, 13(4). https://doi.org/10.18549/PharmPract.2015.04.627

Suter, E., Arndt, J., Arthur, N., Parboosingh, J., Taylor, E., \& Deutschlander, S. (2009). Role understanding and effective communication as core competencies for collaborative practice. Journal of Interprofessional Care, 23(1), 41-51. https://doi.org/10. $\underline{1080 / 13561820802338579}$

Visacri, M.B., Figueredo, I.V., \& de Mendonca Lima, T. (2020). Role of pharmacist during the COVID 19 pandemic: a scoping review. Research in Social and Administrative Pharmacy (EPub ahead of print). https://doi.org/10.1016/j.sapharm.2020.07.003

Wood, V., Flavell, A., Vanstolk, D., Bainbridge, L., \& Nasmith, L. (2009). The road to collaboration: developing an interprofessional competency framework. Journal of Interprofessional Care, 23(6), 621-629. https://doi.org/10.3109/13561820903051477 